\title{
Deliberative Demokratie in der europäischen Mehrebenenpolitik - eine zweite Replik ${ }^{1}$
}

\section{Liberalismus, Kommunitarismus und die aktive Politik}

Habermas beginnt seine Replik mit der Erörterung »liberaler « und »kommunitaristischer " Prämissen der Demokratietheorie und interpretiert meine Position als krypto-kommunitaristisch. Das mag im Kontext der Diskussion so erscheinen, obwohl ich meine Position eher der "republikanischen" Tradition zurechnen würde. ${ }^{2}$ Aber ich denke ohnehin, dass man in normativen Diskursen, die sich auf die Praxis moderner demokratischer Verfassungsstaaten beziehen, Argumente, die aus liberalen, republikanischen oder kommunitaristischen Prämissen abgeleitet sind, zwar unterschiedlich gewichten, aber nicht in ihrer prinzipiellen Relevanz bestreiten kann. Für sich genommen freilich, erscheint mir die liberale Demokratietheorie wegen ihrer Überbetonung der individuellen Selbstbestimmung und konstitutioneller Abwehrrechte gegen freiheitsbeschränkende Politik (die sie mit Lesarten des republikanischen Non-domination-Prinzips teilt ${ }^{3}$ ) in der Tat defizitär. Aber ich zögere auch, Habermas' Charakterisierung des kommunitaristischen Ansatzes mit seiner Fixierung auf einen statischen Wertekonsens zu übernehmen. Aus meiner Perspektive fehlt hier wie dort die Dimension der kollektiven Selbstbestimmung: Demokratie als (wie immer prekäre) Chance der Nutzung staatlicher Handlungsressourcen (Recht, Geld und legitimer Zwang) zur aktiven Gestaltung gesellschaftlicher Verhältnisse, zur Erreichung kollektiver Ziele und zur Abwehr drohender Gefahren.

Aus dieser Perspektive kann ich Habermas' Kritik des kommunitaristischen Modells zustimmen: Ja, die kognitive Wendung der politischen Philosophie ist in Form des Sozialkonstruktivismus auch in der empirischen Politikforschung angekommen. Und obwohl echte Präferenzwechsel nicht leicht von Etikettenwechseln zu unterscheiden sind, haben wir akzeptiert, dass Ideen und die daraus abgeleiteten Argumente nicht nur Zweck-Mittel-Kalküle, sondern auch die Definition subjektiver Interessen und die normativen Wertorientierungen politischer Akteure prägen

1 Jürgen Habermas hat mich freundlicherweise aufgefordert, nach einer Serie von klärenden privaten Briefwechseln seine öffentliche Antwort auf meine Replik noch einmal zu kommentieren. Im Rahmen dieser Korrespondenz habe ich nicht nur die Annäherung der Sichtweisen, sondern auch die diskursive Klärung der eigenen Position als Ergebnis verständigungsorientierter und geduldiger Diskussion sehr schätzen gelernt.

2 Scharpf 2013.

3 So etwa Pettit 2014. 
und verändern können. ${ }^{4}$ Die Frage ist dann, unter welchen Bedingungen und wie schnell mit der von Habermas erwarteten Umorientierung von der nationalen auf die gesamteuropäische normative Rahmung gerechnet werden könnte.

Zuvor aber noch ein Wort zum Zusammenhang mit der Demos-Diskussion. Die aktive, gesellschaftsgestaltende Politik muss in der Lage sein, zur Erreichung kollektiver Ziele die kollektiven Ressourcen des Gemeinwesens einschließlich des staatlichen Gewaltmonopols auch gegen Widerstände einzusetzen. Sie muss also in höherem Maße als der Liberalismus, der die Reichweite der Politik durch Grundrechte und Gewaltenteilung konstitutionell eng beschränken will, ${ }^{5}$ die jeweiligen Grenzen des (rechtlich zulässigen und institutionell möglichen) politischen Handelns im politischen Prozess selbst bestimmen. Mit der potenziell größeren Reichweite und Eingriffstiefe der Politik steigt aber die Wahrscheinlichkeit politischer Interessen- und Richtungskonflikte - und damit steigen auch die Anforderungen an die Legitimität eines politischen Systems, das majoritäre Entscheidungen ermöglicht. Hier liegt, so denke ich, der Kern des No-Demos-Problems einer aktiven und majoritär durchgesetzten aktiven Politik auf der europäischen Ebene.

Welche Gründe aber könnten zur Akzeptanz von Entscheidungen verpflichten, welche die gravierenden Interessen oder normativen Präferenzen einer überstimmten Minderheit (die nicht durch Grundrechte geschützt werden) verletzen? Ich denke nicht, dass dafür die Berufung auf einen Wertekonsens ausreicht. Höhere Legitimationskraft hätte die geteilte Vorstellung gemeinsamer Ziele oder Gefahren, verbunden mit dem reziproken Vertrauen darauf, dass die je eigenen Belange auch als ein Argument in den Nutzenfunktionen der jeweiligen Mehrheit einen Stellenwert haben. ${ }^{6}$ Erst die wechselseitig unterstellte "Einbeziehung des Anderen « (um einen großartigen Habermas-Titel zu verwenden) rechtfertigt die generelle Bereitschaft der Minderheit, auch unerwünschte Entscheidungen der Mehrheit als verbindlich zu akzeptieren. Mehr soll aus meiner Sicht der Demos-Begriff nicht leisten.

Damit ist freilich noch nichts über das empirische Substrat solidarischer Überzeugungen gesagt. Immerhin ist klar, dass sie nicht universell gefordert, ubiquitär unterstellt oder einfach positiv-rechtlich dekretiert werden können. Auch wo sie zunächst vorliegen, kann der Verdacht missbrauchter Mehrheitsherrschaft sie untergraben, ebenso wie umgekehrt der Verzicht auf die Anwendung der Mehrheitsregel den Aufbau wechselseitigen Vertrauens und den politischen Zusammenhalt gespaltener Gesellschaften und sogar eine gesteigerte politische Handlungs- und Gestaltungsfähigkeit nationaler »Konsensdemokratien « ermöglichen kann. ${ }^{7}$

4 Ich kann das jetzt nicht nachprüfen, aber es scheint mir unwahrscheinlich, dass kommunitaristische Autoren ernsthaft behaupten, der Kranz identitätsstabilisierender Hintergrundüberzeugungen solle selbst "gegenüber Änderungen durch demokratische Willensbildung immun sein $«$.

5 Bellamy 2007.

6 Claus Offe hat das so formuliert: "Bevor sie staatliche Autorität anerkennen, müssen die Bürger sich gegenseitig anerkennen, nämlich als hinreichend sgutwillig (vertrauenswürdig) und 'nicht-indifferent ( (solidarisch) " (Offe 1998, S. 105).

7 Lijphart 2012. 
Für die Europäische Union gibt es in der Tat empirische Hinweise darauf, dass die Entwicklung solidarischer Einstellungen unter den europäischen Bürgern in Gang gekommen ist ${ }^{8}$ und auch in der internen Politik der Mitgliedstaaten ist die transnationale Umverteilung durch die EU-Kohäsionsprogramme und die höheren Netto-Beiträge der reicheren Staaten zum EU-Haushalt offenbar nur in Großbritannien ein politisch virulentes Thema. Dennoch halte ich es für riskant, wenn Habermas im Vorgriff auf die - mögliche (!) - weitere Stärkung kooperativer Einstellungen schon jetzt die Bewältigung der Euro-Krise durch eine solidarische europäische Politik ins Auge fasst. Selbst wenn diese pragmatisch wirksam wäre (was ich in meiner Replik verneint habe), könnte die majoritäre Überforderung einer schwachen Solidaritätsbereitschaft einen politischen Rückschlag zur Folge haben, der andernfalls mögliche Fortschritte der sozialen Integration blockieren oder auch umkehren könnte.

Aber unabhängig von dieser empirischen Einschätzung politischer Risiken sehe ich drei theoretische Schwierigkeiten, die dem Vorschlag einer solidarischen und majoritären Überwindung der gegenwärtigen Integrationskrise entgegenstehen. Die erste betrifft das Verhältnis zwischen der Union und ihren Mitgliedern, die zweite das Verhältnis zwischen den Bürgern der Mitgliedstaaten und die dritte das Verhältnis zwischen öffentlicher Diskussion und deliberativen Entscheidungsprozessen auf der europäischen Ebene.

\section{Noch einmal: Legitime Diversität}

Im Hinblick auf mein Plädoyer für die Respektierung legitimer Diversität zwischen den EU-Staaten, die Habermas als »kulturellen Naturschutz [für] parochiale Formen des Kapitalismus « ablehnt, bleibt der Dissens bestehen, aber es ist deutlich geworden, dass er seinen Grund in dem Kriterium der »transnationalen Verallgemeinerung von Interessen « findet, das Habermas für die wahrheitsorientierte Deliberation auf europäischer Ebene postuliert. Dieses negiert in der Tat die von mir ins Feld geführten Argumente für demokratietheoretische Grenzen legitimer Mehrheitsentscheidungen.

Dabei hat Habermas Recht: Die Verteidigung der Varianten eines demokratisch domestizierten Kapitalismus kann nicht mit einer Analogie zum konstitutionellen Schutz universeller Individualrechte oder von Gruppenrechten religiöser, sprachlicher oder ethnischer Minderheiten begründet werden. Hier geht es jedenfalls nicht in erster Linie um die individuellen Interessen und Rechte von Bürgern oder von Gruppen ${ }^{9}$ (also etwa um die Koalitionsfreiheit, das Streikrecht oder das Wahlrecht). Sondern es geht um die Verteidigung historisch erkämpfter gesellschaftlicher Arrangements und staatlicher Regimes, in denen der Ausgleich zwischen den antagonistischen Interessen von Kapital, Arbeit und Staat (das heißt den Interessen an steuerfinanzierten öffentlichen Gütern, Dienstleistungen und Sozialtransfers) jeweils bis

8 Risse 2014.

9 Hier habe ich in meiner ersten Replik nicht klar unterschieden. 
auf Weiteres mit den Mitteln des staatlichen Rechts fixiert wird. Diese hatten ihre Gestalt in den Nachkriegsjahrzehnten unter dem Schutz des internationalen Regimes eines "embedded liberalism " ${ }^{10}$ gefunden. Und sie sind parochial, weil sie unter je besonderen ökonomischen, sozialen und institutionellen Bedingungen durch Klassenkämpfe, politische Konflikte und historische Kompromisse geschaffen wurden.

Diese institutionalisierten Interessen-Balancen könnten auf europäischer Ebene schon deshalb nicht reproduziert werden, weil die sie jeweils ermöglichenden Machtverhältnisse und strategischen Optionen dort nicht ebenso vorhanden wären. Aber selbst wenn in der europäischen Deliberation Machtkalküle gar keine Rolle spielten und Entscheidungen allein durch die transnationale Verallgemeinerung von Interessen bestimmt würden, hätten sozialstaatliche Lösungen kaum eine Chance. Der Grund liegt darin, dass (wenn man effektive Kartellverbote voraussetzt) die im Prinzip einheitlichen Interessen der Kapitalseite ${ }^{11}$ an Deregulierung, Liberalisierung und Steuersenkung jedenfalls im Lichte der dominanten ökonomischen Theorie und der Rechtsprechung des Europäischen Gerichtshofs zugleich auch als uneingeschränkt generalisierungsfähig erscheinen. Demgegenüber würden Argumente zur Verteidigung parochialer Institutionen nach den Habermas'schen Diskursregeln sogleich disqualifiziert, und auch Versuche einer argumentativen Verallgemeinerung der Interessen von Arbeitnehmern und Staatsklienten an den Errungenschaften ihrer jeweils bestehenden Regimes würden durch Konflikte zwischen rumänischen Wanderarbeitern, deutschen Niedriglöhnern, spanischen Arbeitslosen und dänischen Steuerzahlern beeinträchtigt. Kurz, auch eine durch das Prinzip der deliberativen Verallgemeinerung disziplinierte europäische Politik müsste weiterhin die neoliberale Variante der kapitalistischen politischen Ökonomie institutionalisieren. ${ }^{12}$

Vor allem aber ignoriert Habermas hier den normativen Eigenwert der europäischen Mehrebenen-Demokratie: Bei dem "Naturschutz " ginge es ja nicht nur um die Interessen jeweils begünstigter Bürger, sondern auch um die "normativen Errungenschaften « politisch (und im Prinzip auch deliberativ) legitimierter Selbstregierung in den Mitgliedstaaten. Sie sind gewiss nicht gegen die Notwendigkeit von Veränderungen geschützt. Aber diese können nicht mit leichter Hand durch (rein output-orientierte!) Hinweise auf die Vorteile einer »hypothetisch angenommenen Steigerung der Produktivität « legitimiert werden. Änderungen müssen, wie der jahrelange Streit um die deutschen Hartz-IV-Reformen gezeigt hat, im nationalen Kontext politisch erkämpft und verantwortet werden. Werden sie europäisch oktroyiert, sei es von der Troika oder von einer Mehrheit anderer Europäer im Europäischen Parlament, zerstören sie die Demokratie in den Mitgliedstaaten, auf der die Legitimität der Europäischen Union nach wie vor aufbauen muss.

10 Ruggie 1982.

11 Offe, Wiesenthal 1980.

12 Scharpf 2010. 


\section{Zur Begründung transnationaler Solidarität}

Habermas hat in seinem Aufsatz und auch in seiner Replik gefordert, dass bei der Bewältigung der Euro-Krise die "unter deutscher Führung anderen Ländern oktroyierte Politik der inneren Abwertung durch eine solidarisch vereinbarte Politik " abzulösen sei. Dabei soll es, so unterstelle ich, um die Überwindung der Wirtschaftsund Sozialkrisen in den sogenannten Schuldnerländern gehen und nicht lediglich um den pünktlichen Schuldendienst, den die derzeitigen "Rettungskredite" ja sicherstellen. ${ }^{13}$ Habermas' Forderung impliziert dann die pragmatische Frage, ob eine solidarische europäische Politik unter den Bedingungen der Währungsunion diese Krisen bewältigen könnte (die ich verneint habe). Und sie impliziert die normative Frage nach der Rechtfertigung einer transnationalen Umverteilung zugunsten der Krisenländer.

Ein europaweiter Gerechtigkeitsdiskurs könnte diese Rechtfertigung jedenfalls nicht leisten. Er müsste in erster Linie bitterarme Staaten außerhalb der Euro-Zone wie Bulgarien und Rumänien begünstigen. Und selbst wenn es nur um die EuroZone ginge, kämen zunächst die osteuropäischen Mitgliedstaaten an die Reihe, in denen das Pro-Kopf-Einkommen unter dem in Griechenland liegt (um von Irland gar nicht zu reden). ${ }^{14}$ Mit welchen Argumenten aber könnten dann die Bürger in Deutschland, Finnland, Lettland oder der Slowakei von ihrer Pflicht zur solidarischen Unterstützung gerade der gegenwärtigen Krisenländer überzeugt werden?

Als ein mögliches Muster kämen dafür die Argumente in Betracht, mit denen nach der deutschen Vereinigung die immensen West-Ost-Transfers in einer Weise begründet wurden, die auch moralisch gefestigte westdeutsche Verfassungspatrioten, die alle völkischen oder national-kulturellen Identitätszumutungen hinter sich gelassen hatten, nicht ablehnen konnten. Sie liefen letztlich auf ein einfaches, aber moralisch unabweisbares Argument hinaus, das auf einen Satz reduziert werden kann: "Die West- und die Ostdeutschen haben den Krieg gemeinsam angefangen und gemeinsam verloren; also müssen sie auch die Folgen solidarisch tragen «. Nach der von mir vertretenen Einschätzung der institutionellen und ökonomischen Ursachen der Euro-Krise hätte ein äquivalentes, dem juristischen Modell der »Haftung für vorausgegangenes Tun « nachgebildetes moralisches Argument auch für eine solidarische Euro-Krisenpolitik formuliert werden können: "Der Euro war von Anfang an ein ökonomisch verfehltes frivoles Experiment (Wolfgang Streeck). Wir haben jedoch die monetäre Über-Integration im gemeinsamen Irrtum beschlossen und so auch die Krise gemeinsam verursacht. Und obwohl nun die einen Länder sich als Krisengewinnler und die anderen als Krisenopfer wiederfinden, sind wir doch mora-

$13 \mathrm{Da}$ die griechische Staatsschuld inzwischen weitgehend von privaten auf öffentliche Gläubiger (IMF, EZB und Rettungsfonds) übergegangen ist, fließen Rettungskredite unmittelbar an diese zurück. Zugleich verlängern sie aber die Abhängigkeit der Kreditnehmer von den »Reform «-Auflagen der Gläubiger - was sowohl die griechische Forderung nach einem Schuldenschnitt als auch dessen rigorose Ablehnung durch den deutschen Finanzminister gut erklärt.

14 Vgl. FAZ 2015. 
lisch verpflichtet, den gemeinsamen Fehler gemeinsam zu korrigieren und seine Folgen solidarisch zu bewältigen «.

Dieses kognitiv basierte moralische Argument, das die Verantwortung auf die Staaten der Euro-Zone begrenzt und eine spezifische Solidarität mit den Krisenopfern begründet hätte, war freilich in der Diskussion über die Euro-Rettung ohne Chance. Berlin, Brüssel und Frankfurt einigten sich schnell auf eine objektiv falsche, ${ }^{15}$ ja geradezu perfide und transnationale Konflikte schürende Erklärung, die die Schuld an der Krise allein der verantwortungslosen Fiskalpolitik der Krisenstaaten zuschrieb, die man aber zur Verteidigung des Euro leider trotzdem vor dem Bankrott retten müsse.

Aber auch Habermas könnte das hier vorgeschlagene moralische Argument nicht zur Begründung seiner Forderung einsetzen, weil er ja an der Währungsunion als europäischer Errungenschaft festhalten will. Wenn diese aber im Prinzip richtig oder nur der Verzicht auf die politische Union (die meines Erachtens wenig geändert hätte) ein Fehler war, ${ }^{16}$ dann kann solidarische Haftung auch nicht mit der originären Täter-Verantwortung für die monetäre Über-Integration begründet werden. Habermas kann deshalb - ebenso wie die wenigen deutschen und vielen ausländischen Keynesianer unter den Ökonomen - die Stoßrichtung seiner Kritik nur gegen die Euro-Rettungspolitik und deren Verteilungsfolgen richten. Über deren ökonomische Wirkung und sozio-ökonomische Kosten-Nutzen-Bilanz ist jedoch eher mit pragmatischen als mit grundsätzlich-moralischen Argumenten zu streiten.

Für die von Habermas geforderte solidarische Bewältigung der Krise liegt darin ein "diskurspolitischer « Nachteil. Denn wenn es denn gelänge, diese Forderung auf die europäische Agenda zu setzen, fehlte ein generalisierungsfähiges moralisches Argument, mit dem die Befürworter einer "Transferunion" der zu erwartenden Eskalation transnationaler Verteilungskonflikte und der Mobilisierung nationaler Feindbilder und wechselseitiger Anschuldigungen entgegentreten könnten.

\section{Die prekäre Arbeitsteilung zwischen Öffentlichkeit und deliberativer Entscheidung}

Mein letzter Kommentar betrifft Habermas' - möglicherweise missverstandene Antwort auf das von mir unterstellte Spannungsverhältnis zwischen seiner auf vernünftigen Konsens zielenden Theorie der deliberativen Politik und seinem Plädoyer für konflikthafte öffentliche Auseinandersetzungen über europäische Politik. Er schreibt: "Die legitimerende Kraft des demokratischen Verfahrens geht auf die Verbindung von inklusiver Beteiligung mit Deliberation zurück « (Hervorhebung im Original), und er interpretiert diese Verbindung als »institutionelle Arbeitsteilung zwischen den informellen Kommunikationen in der Öffentlichkeit und qualifizier-

15 Scharpf 2011.

16 Dagegen könnten Autoren wie Claus Offe oder Henrik Enderlein, die die Schaffung des Euro zwar für einen schrecklichen Fehler halten, der aber wegen befürchteter Änderungskosten nicht revidiert werden kann (so zum Beispiel Offe 2015), widerspruchsfrei für eine solidarische Krisenbewältigung eintreten. 
ten Beratungsverfahren in den rechtsetzenden [...] Institutionen «, die er dann in Fußnote 10 noch schärfer akzentuiert: "Die informelle Kommunikation in der breiten Öffentlichkeit kann [...] auch die in robusten oder gar wüsten Formen ausgetragenen Konflikte aushalten. Erst in den deliberierenden und beschließenden Körperschaften muss zwischen konkurrierenden Positionen mit Mehrheit entschieden werden «. Und: »Diese antagonistische Dynamik kann für die Mobilisierung relevanter öffentlicher Meinungen und daher für den Willensbildungsprozess im Ganzen durchaus funktional sein " (Hervorhebung im Original).

Habermas erwartet also, so interpretiere ich diese Klarstellung, dass zwar die in den wüsten Konflikten zu Tage tretende Information, nicht aber die antagonistische Dynamik in die Sphäre der institutionellen Deliberation hineinwirken werde. Was den moderaten Stil parlamentarischer Beratungen angeht, mag dies zutreffen. Aber in der Realität der repräsentativen Demokratie sind die theoretisch abgegrenzten Sphären der politischen Kommunikation durch allgemeine Wahlen, die Konkurrenz politischer Parteien, Meinungsbefragung, Medien und den Mechanismus der antizipierenden Reaktion eng aneinander gekoppelt. Überdies werden ja auch die wüsten Konflikte im informellen Vorfeld zu einem erheblichen Teil von den Parteien der Regierung und der Opposition bestritten, und ihr Ausgang kann die Mehrheitsverhältnisse in den deliberierenden und beschließenden Körperschaften bestimmen. Sollen und können diese dann über die dominante Tendenz der öffentlichen politischen Auseinandersetzung hinweggehen?

In der Politikwissenschaft wird diese Frage als Spannungsverhältnis zwischen input-orientierter responsiveness und output-orientierter responsibility demokratischer Politik interpretiert, ${ }^{17}$ wobei die normative Antwort im Prinzip nicht strittig ist: Regierung und Parlament sind durch Amtseid auf das Gemeinwohl verpflichtet. Deshalb könnte eine "populistische", also nicht durch deliberative Beratung geprüfte und validierte majoritäre Entscheidung nicht »als vernünftige Grundlage einer gemeinsamen Praxis « ${ }^{18}$ Legitimität beanspruchen. Aber zugleich muss auch die deliberativ veredelte Politik in allgemeinen Wahlen politisch verantwortet werden - und der Preis für unpopuläre Entscheidungen soll und kann sehr hoch sein, wie Schröder und die rot-grüne Koalition nach den Hartz-Reformen erfahren haben. Deshalb kann, wo diese Rückkoppelung majoritärer Entscheidungen an die potenzielle Sanktionsgewalt der egalitären Wahl in der Theorie ignoriert wird (oder wie in der Europäischen Union faktisch fehlt), die Deliberation in den beschließenden Institutionen vielleicht die Output-Qualität verbessern, jedoch nicht demokratische

\section{Mair 2013.}

18 So Jürgen Habermas: "Wegen des internen Zusammenhangs mit einer deliberativen Praxis begründet die Mehrheitsregel die Vermutung, daß die fallible Mehrheitsmeinung bis auf weiteres, nämlich bis die Minderheit die Mehrheit von der Richtigkeit ihrer Auffassung überzeugt hat, als vernünftige Grundlage einer gemeinsamen Praxis gelten darf « (Habermas 1992, S. 371). Das Mehrheitsvotum ist hier also nicht konstitutiv, sondern lediglich indikativ für die Legitimität von Politik. Das Argument erinnert an das (schon von Rousseau antizipierte) Condorcet-Jury-Theorem: Wenn es in der Politik um Wahrheit geht und wenn alle sich um die Erkenntnis der Wahrheit bemühen, dann begründet die größere Zahl der Voten die Vermutung der Richtigkeit (zur Kritik: Ladha 1992). 
Legitimität vermitteln. ${ }^{19}$ Auch die postulierte Arbeitsteilung zwischen den informellen und formalen Sphären des deliberativen Modells könnte das für die Legitimität der repräsentativen Demokratie konstitutive Spannungsverhältnis zwischen responsiveness und responsibility nicht aufheben.

Im demokratischen Nationalstaat mag das Pendel zeitweilig in die eine oder andere Richtung ausschlagen. In der Europäischen Union dagegen fehlen bisher die institutionellen und politischen Voraussetzungen für die Verbindung der beiden Sphären. Der europäischen Politik fehlt die legitimierende Rückkoppelung an die Sanktionsgewalt europäischer Wähler; und auch die auf europäischer Ebene locker verbundenen »Parteifamilien « tragen nicht zu einem effektiven »kommunikativen Diskurs ${ }^{20}$ zwischen politischen Akteuren und den noch überwiegend nationalen Öffentlichkeiten bei.21 Und für die von Habermas postulierte »Verbindung von inklusiver Beteiligung mit Deliberation « reicht es ja nicht, wenn nur der politisch isolierte »koordinierende Diskurs « unter den Akteuren der europäischen Ebene deliberative Qualität erreichen kann. ${ }^{22}$

Um dem Ziel einer demokratisch legitimierten majoritären europäischen Politik näherzukommen, muss Habermas die Politisierung europäischer Optionen verlangen. Und im Hinblick auf die Bewältigung der gegenwärtigen Krise setzt er den Warnungen vor einer Eskalation politischer Konflikte die Hoffnung auf eine Änderung des politischen Interpretationsrahmens entgegen: Statt der gegenwärtig dominanten nationalen Zurechnung ökonomischer Systemprobleme soll die politische Auseinandersetzung die Interessenlagen der "Krisengewinnler und -verlierer jeweils über nationale Grenzen hinweg verbinden « und damit zugleich die demokratische Legitimität europäischer Mehrheitsentscheidungen stärken. Im politisch isolierten Europäischen Parlament könnte diese »klassenpolitische « Änderung des politischen Interpretationsrahmens der Euro-Krise vielleicht sogar gelingen. Aber was wäre »unter der hypothetischen Voraussetzung eines funktionsfähigen, das heißt mit den nationalen Öffentlichkeiten rückgekoppelten « Parlaments zu erwarten? Vielleicht ließen sich - aus den oben erörterten Gründen - auch hier die Interessen der Kapitalseite transnational einheitlich mobilisieren. Aber wie stünde es um die Interessen der Lohnsteuerzahler und Sozialklienten, der Beschäftigten und der Arbeitslosen in Lettland und Österreich, Irland und Slowenien, Finnland und Portugal oder eben in Griechenland und Deutschland? Würde nicht gerade der Versuch, einen im Europäischen Parlament vielleicht definierbaren Klassenkonflikt zur Mobilisierung politischer Öffentlichkeiten in den Mitgliedstaaten einzusetzen, die transnationalen

19 Gaus 2013.

20 Vivien A. Schmidt unterscheidet zwischen dem coordinative discourse unter den Entscheidungsbeteiligten und dem communicative discourse zwischen diesen und der politischen Öffentlichkeit, und sie betont die institutionell begründete faktische Beschränkung auf den ersteren in der europäischen Politik (Schmidt 2002, S. 230-250).

21 Im letzten Europawahlkampf jedenfalls haben weder die "Spitzenkandidaten « noch das deutsche Kartell der pro-europäischen Parteien die Optionen der Euro-Rettungspolitik thematisiert.

22 Buchstein, Jörge 2003. 
Konflikte auf der »linken « Seite des politischen Spektrums eskalieren lassen? Weder Habermas noch ich könnten das ausschließen.

Ich habe aus unserer Diskussion viel gelernt, aber ich bin in empirisch-pragmatischer Hinsicht noch nicht überzeugt: Habermas geht es um die Weiterentwicklung der Europäischen Union zu einer politisch handlungsfähigen "demokratischen und zugleich überstaatlichen Föderation " auf der Basis des verfassungstheoretischen Konzepts der doppelten Souveränität und des politiktheoretischen Konzepts der deliberativen Demokratie. Zugleich sollen seine Argumente aber auch den Weg zu einer effektiven und demokratisch legitimierten Bewältigung der akuten Wirtschafts- und Sozialkrise in der Euro-Zone weisen. Die Dringlichkeit dieser zweiten Absicht erfordert jedoch Akzentsetzungen und Verzichte in der anwendungsorientierten Argumentation, die mir eher kritisierbar erscheinen als die Theorie selbst.

So muss Habermas, um die Vetomacht der nationalen Regierungen gegen die favorisierte Politik abzubauen, alle institutionellen Vorkehrungen zur Bewahrung der kulturellen, institutionellen und polit-ökonomischen Vielfalt Europas ${ }^{2.3}$ als »kulturellen Naturschutz « disqualifizieren, obwohl eine weitere Entfaltung seines Konzepts der doppelten Souveränität die Suche nach zugleich autonomieschonenden und gemeinschaftsverträglichen Lösungen eher nahelegen würde. Überdies verzichtet er, weil er die Währungsunion als Fortschritt der supranationalen Integration nicht infrage stellen will, auf eine moralisch tragfähige Begründung für die solidarische Bewältigung der Euro-Krise. Und schließlich bleiben auch die realen Voraussetzungen unklar, die erfüllt sein müssten, ehe die europäische Krisenpolitik sich auf die - für Habermas' Theorie der deliberativen Demokratie legitimationsbegründende - Verbindung von inklusiver Beteiligung und deliberativer Entscheidung berufen dürfte. Aber dies sind Einwände, die sich nicht gegen diese Theorie richten, sondern gegen deren zu wenig vermittelte Verwendung zur Bewältigung der akuten europäischen Krise.

\section{Literatur}

Abelshauser, Werner 2007. Europa in Vielfalt einigen: Eine Denkschrift. Universität Bielefeld.

Bellamy, Richard 2007. Political constitutionalism: a republican defense of the constitutionality of democracy. Cambridge: Cambridge University Press.

Buchstein, Hubertus; Jörge, Dirk 2003. „Das Unbehagen an der Demokratietheorie ", in Leviathan 31, 4, S. 470-495.

FAZ 2015. "Zähneknirschen über Griechenland in Osteuropa «, in Frankfurter Allgemeine Zeitung vom 28. Februar 2015, S. 20.

Gaus, Daniel 2013. "Qualität statt Partizipation und Gleichheit? Eine Bemerkung zum epistemischen Sinn von Demokratie ", in Leviathan 41, 2, S. 264-290.

Habermas, Jürgen 1992. „Deliberative Politik - ein Verfahrensbegriff der Demokratie «, in Jürgen Habermas: Faktizität und Geltung. Beiträge zur Diskurstheorie des Rechts und des demokratischen Rechtsstaats, S. 349-398. Frankfurt a. M.: Suhrkamp.

Ladha, Krishna K. 1992. "The condorcet jury theorem, free speech and correlated votes ", in American Journal of Political Science 36, 3, S. 617-634.

Lijphart, Arend 2012. Patterns of democracy: government forms and performance in 36 countries. New Haven: Yale University Press. 
Mair, Peter 2013. Ruling the void: the hollowing of Western democracy. London: Verso.

Offe, Claus 1998. "Demokratie und Wohlfahrtsstaat: Eine europäische Regimeform unter dem Streß der europäischen Integration", in Internationale Wirtschaft, nationale Demokratie, hrsg. v. Streeck, Wolfgang, S. 99-136. Frankfurt a. M.: Campus.

Offe, Claus 2015. Europe entrapped. Cambridge: Polity Press.

Offe, Claus; Wiesenthal, Helmut 1980. "Two logics of collective action: theoretical notes on social class and organizational form ", in Political Power and Social Theory 1, S. 67-115.

Pettit, Philip 2014. Just freedom. A moral compass for a complex world. New York: Norton.

Risse, Thomas 2014. "No demos? Identities and public spheres in the Euro crisis", in Journal of Common Market Studies 52, 6, S. 1207-1215.

Ruggie, John G. 1982. "International regimes, transactions and change: embedded liberalism in the postwar economic system ", in International Organization 36, 2, S. 379-415.

Scharpf, Fritz W. 2010. "The asymmetry of European integration, or why the EU cannot be a social market economy ", in Socio-economic Review 8, 2, S. 211-250.

Scharpf, Fritz W. 2011. "Monetary Union, fiscal crisis and the pre-emption of democracy ", in Journal for Comparative Government and European Policy 9, 2, S. 163-198.

Scharpf, Fritz W. 2013. "Legitimacy intermediation in the multilevel European polity and its collapse in the Euro crisis ", in Staatstätigkeiten, Parteien, Demokratie. Festschrift für Manfred G. Schmidt, hrsg. v. Armingeon, Klaus, S. 567-596. Wiesbaden: Springer VS.

Schmidt, Vivien E. 2002. The futures of European capitalism. Oxford: Oxford University Press. 
Zusammenfassung: Auch nach Habermas’ klärender Replik bleiben noch Differenzen. Sie betreffen die Legitimität majoritärer Politik in der gegenwärtigen Europäischen Union, die Anwendung des Prinzips der transnationalen Verallgemeinerung auf Vorkehrungen zum Schutz der institutionellen, kulturellen und sozioökonomischen Vielfalt der Mitgliedstaaten und die realen Voraussetzungen der deliberativen Demokratie im europäischen Kontext.

Stichworte: Europäische Union, deliberative Demokratie, Majoritätsregel, Euro-Krise

\section{Deliberative democracy in the multilevel European polity - a second reply}

Summary: In spite of Habermas' clarifying response, differences remain regarding the legitimacy of majority rule in the present European Union, in particular to institutional protections for the institutional, cultural and socio-economic diversity of EU member states and to the preconditions of deliberative democracy in the European context.

Keywords: European Union, deliberative democracy, majority rule, Euro crisis

\section{Autor}

Prof. Fritz W. Scharpf

Max-Planck-Institut für Gesellschaftsforschung

Paulstr. 3

$50676 \mathrm{Köln}$ 